\title{
Erratum
}

\section{Erratum to: Asymptotics and analytic modes for the wave equation in similarity coordinates}

\author{
ROLAND DONNINGER
}

\section{Erratum to: J. Evol. Equ. 9 (2009), 511-523 DOI 10.1007/s00028-009-0022-x}

In the original publication of the article, the use of orthogonal projections is incorrect since they do not commute with the semigroups in question. However, the problem is easily fixed by using the correct Riesz projections instead. The results remain unchanged.

\section{The free equation}

The projection $P$ on p. 517 needs to be constructed as follows. For each analytic eigenvalue $\lambda_{j}, j=1,2, \ldots, 2 k$, with (normalized) eigenfunction $\mathbf{u}\left(\cdot, \lambda_{j}\right)$, one defines

$$
P_{j} \mathbf{f}:=\frac{f_{1}^{(j-1)}(1)+f_{2}^{(j-1)}(1)}{\gamma_{j}} \mathbf{u}\left(\cdot, \lambda_{j}\right)
$$

where $\gamma_{j}:=u_{1}^{(j-1)}\left(1, \lambda_{j}\right)+u_{2}^{(j-1)}\left(1, \lambda_{j}\right)$ (it follows from the explicit form of $\mathbf{u}\left(\cdot, \lambda_{j}\right.$ ) given in the proof of Lemma 3 that $\gamma_{j} \neq 0$ ). By Sobolev embedding, one sees that $P_{j}$ is bounded on $\mathcal{H}^{2 k}$ and obviously, $P_{j}^{2}=P_{j}$. Furthermore, it is straightforward to see that $P_{j}$ commutes with $L_{0}$. Then, one sets $P:=\sum_{j=1}^{2 k} P_{j}$ which gives the correct (nonorthogonal) projection that commutes with the semigroup $S_{0}(\tau)$. Subsequently, the space $\mathcal{N}^{\perp}$ needs to be replaced by $\operatorname{rg}(1-P)$. 


\title{
2. The semilinear wave equation
}

Similarly, the orthogonal complement $\mathcal{N}^{\perp}$ on p. 520 needs to be replaced by rg (1$P$ ) where $P$ is constructed as follows. Since the perturbation $\left.L^{\prime}\right|_{\mathcal{H}^{2 k}}$ is compact, each analytic eigenvalue $\lambda_{j}^{ \pm}$with $\operatorname{Re} \lambda_{j}^{ \pm}>\frac{1}{2}-2 k$ is isolated, and thus, there exists an associated Riesz projection $P_{j}^{ \pm}$that commutes with the semigroup $S(\tau)$. From the commutator relation in Lemma 1, it follows that $\operatorname{dim} \operatorname{rg} P_{j}^{ \pm}=1$ and one sets $P:=\sum_{j} P_{j}^{ \pm}$. An updated version of the original article can be found on the arXiv preprint server [1].

\section{Reference}

[1] Donninger, Roland. Asymptotics and analytic modes for the wave equation in similarity coordinates. http://arxiv.org/abs/0809.5177.

\author{
R. Donninger \\ Faculty of Physics, Gravitational Physics, \\ University of Vienna, \\ Boltzmanngasse 5, 1090 Vienna, Austria \\ E-mail: roland.donninger@univie.ac.at
}

